\title{
Photouncaging of Carboxylic Acids from Cyanine Dyes with Near-Infrared Light
}

\author{
Hana Janeková, ${ }^{\dagger}$ Marina Russo, ${ }^{\dagger}$ Peter Štacko, ${ }^{\dagger, *}$ \\ ${ }^{\dagger}$ Department of Chemistry, University of Zurich, Wintherthurerstrasse 190, CH-8057 Zurich, \\ Switzerland
}

\begin{abstract}
Near-infrared light (NIR; 650-900 $\mathrm{nm}$ ) as biocompatible stimulus offers unparalleled advantages in terms of availability, high precision in time and space and deep tissue penetration. The development of photocages that operate in this region represents a fundamental challenge due to low energy of the excitation light. Herein, we

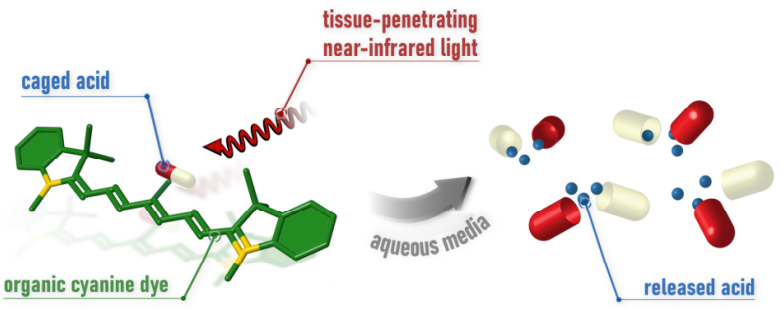
repurpose cyanine dyes into photocages that are available on a multigram scale in three steps and efficiently release carboxylic acids in aqueous media upon irradiation with NIR light up to $820 \mathrm{~nm}$. The photouncaging process is examined using several independent techniques and we provide compulsive evidence that it proceeds via photooxidative pathway. In vitro biocompatibility of the photocages and their photoproducts is also demonstrated. In combination with modularity of the cyanine scaffold, the realization of these accessible photocages will aid fully unleashing the potential of the emerging field of NIR-photoactivation and facilitate its widespread adoption outside the photochemistry community.
\end{abstract}

\section{Introduction}

Photocages are light-sensitive groups that take advantage of biorthogonality as well as unparalleled spatial and temporal resolution of light as a stimulus to unmask and restore activity of substrate. ${ }^{1}$ Particularly valued in biological context, they have been used to activate proteins, ${ }^{2,3}$ nucleotides, ${ }^{4,5}$ drugs $^{6,7}$ and other biologically relevant molecules. ${ }^{8-10}$ However, extending their application towards therapeutic utility is limited by the wavelengths applicable in body and requires shifting their absorption into near-infrared (NIR) phototherapeutic window $(650-900 \mathrm{~nm}) .{ }^{11,12}$

While an impressive collection of organic photocages that can be excited by UV and visible light has been developed in the past decades, ${ }^{13}$ the development of organic, NIR light-activated photocages constitutes a significant challenge in contemporary photochemistry. ${ }^{14}$ Major contributing factors are low energy of the NIR photons, efficient competing deactivation pathways in the excited states and requirement to operate in aqueous media. Consequently, their existence is currently limited to only few examples (Figure 1). 
State-of-the-art NIR photocages
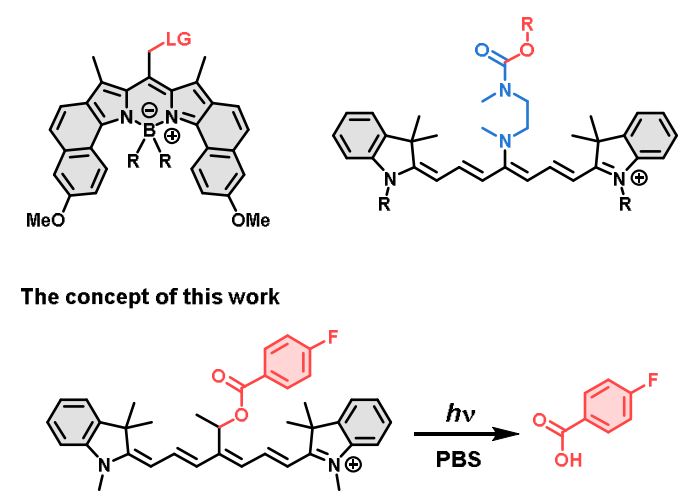

Figure 1. Previously reported NIR-absorbing photocages (top) and the concept of this work (bottom). The caged moiety is depicted in red.

Silicon phthalocyanine was shown to release axial phenolic ligands via photoinduced electron transfer in hypoxic conditions. ${ }^{15}$ Schnermann and co-workers harnessed photooxidative cleavage of cyanines (Cy7) to initiate a reaction cascade releasing phenols, ${ }^{16-18}$ but the required linkers preclude release of other groups e.g. carboxylates, amines. Their application in tumor ${ }^{19,20}$ and traumatic brain injury ${ }^{21}$ treatments lends credibility to the use of singlet oxygen-mediated processes in therapeutic settings. Winter and Weinstain independently reported BODIPY photocages with strong green absorption ${ }^{22,23}$ that have been since extensively optimized to improve the photoreleasing efficiencies ${ }^{24,25}$ and extend their absorption maxima towards the edge of NIR region. ${ }^{26}$ Recently, they were utilized to control oscillations of cardiomyocetes using red light. ${ }^{27}$ Despite significant progress in their design,${ }^{28}$ BODIPY photocages are troubled by lengthy and low yielding synthesis. On the other hand, theory predicts that analogous release is achievable from $\mathrm{Cy} 3$ and Cy7, which share equivalent characteristics of frontier molecular orbitals with BODIPY, ${ }^{29,30}$ which led us to explore this concept.

Here, we describe heptamethine cyanine dyes into a class of easily accessible photocages that efficiently release carboxylic acids in aqueous media upon irradiation with near-infrared light up to $820 \mathrm{~nm}$ (Figure 1). A major asset of these photocages is the fact that the caged functionality is attached directly to the cyanine chromophore without additional linkers. Not only this significantly reduces the complexity of the release process, but it also extends the scope of caged functionalities to those previously beyond the reach, especially for cyanine photocages (i.e. carboxylates). This particular feature promises high tolerance of our system towards the type of caged moiety, limited only by the quality of the leaving group. 


\section{Results and Discussion}

The photocages were synthesized in 3 steps utilizing our recently developed strategy using ring-opening of Zincke salts. ${ }^{31}$ The synthesis started by esterification of carboxylic acids $\mathbf{3 a}-\mathbf{c}$ with $\mathbf{2}$ using EDC.HCl and DMAP in very good to excellent yields (Figure 2). The pyridines $4 \mathbf{a}-\mathbf{f}$ were then transformed to the corresponding Zincke salts 5a-f by a reaction with 2,4-dinitrophenyl tosylate (DNP) in acetone. Subsequently, 5a-f were subjected to a one-pot condensation with indolinium-based heterocycles $\mathbf{6 a - c}$ and AcOK to provide the final photocages 1a-f containing diverse caged carboxylic acids. The methoxysubstituted heterocycle $\mathbf{6 c}$ facilitated preparation of a fully water-soluble derivative 1d, otherwise inaccessible due to poor reactivity of the des-methoxy analogue of $\mathbf{6 c}$. To demonstrate the practical utility of our protocol, we upscaled the synthesis and successfully prepared $\sim 2 \mathrm{~g}$ of the photocage 1a in a single batch without any detrimental effects on the yield.
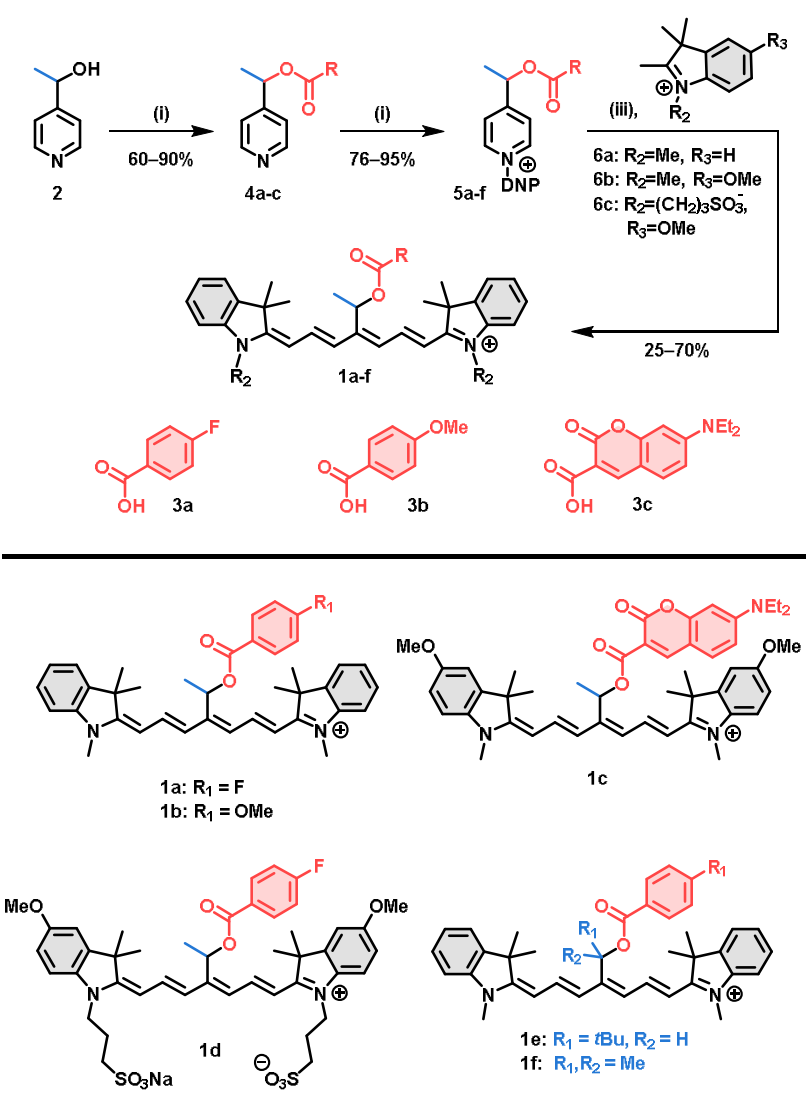

Figure 2. Synthesis of the photocages. (i) 3a-c, EDC.HCl, DMAP, $\mathrm{CH}_{2} \mathrm{Cl}_{2}$. (ii) DNP-OTs, acetone, $40^{\circ} \mathrm{C}$. (iii) AcOK, EtOH, rt. Caged acids are depicted in red.

UV-Vis absorption spectra of the photocages 1a-f in PBS (pH 7.4, $10 \mathrm{mM})$ display intense absorption bands in the NIR region at $\lambda_{\mathrm{em}} \sim 786 \mathrm{~nm}$, typical for the heptamethine cyanine dyes (Table 1 and Figure 2A). ${ }^{32}$ It is worth noting that their absorption maxima nearly perfectly match the wavelengths of commercial diode lasers. The compounds $\mathbf{1} \mathbf{a}-\mathbf{b}$ also exhibit near-infrared emission with the Stokes shifts $(\sim 23 \mathrm{~nm})$ and quantum yields comparable to that of ICG. 
Table 1. Photophysical and Photochemical Properties of 1a-f.

\begin{tabular}{|c|c|c|c|c|c|}
\hline & $\lambda_{\mathrm{abs}} / \mathrm{nm}^{a}$ & $\lambda_{\mathrm{em}} / \mathrm{nm}^{b}$ & $\mathcal{E}_{\max }{ }^{a, c}$ & $\Phi_{\mathrm{F}} / \%^{b}$ & $\Phi_{\mathrm{dec}} \times 10^{5 a, e}$ \\
\hline $1 \mathrm{a}$ & 786 & 809 & 155400 & $2.9 \pm 0.7$ & $2.2 \pm 0.5$ \\
\hline $1 b$ & 786 & 808 & 176400 & $3.1 \pm 0.1$ & $2.6 \pm 0.1$ \\
\hline $1 c$ & 808 & 844 & 57400 & $<2 \%^{d}$ & $46.8 \pm 6.6$ \\
\hline $1 d$ & 817 & 860 & 123200 & $<2 \%^{d}$ & $1.7 \pm 0.3$ \\
\hline le & 796 & 826 & 107100 & $<2 \%^{d}$ & $5.4 \pm 0.4$ \\
\hline If & $806^{b}$ & 843 & $42000^{b}$ & $<2 \%^{d}$ & $1.3 \pm 0.3^{b}$ \\
\hline \multicolumn{6}{|c|}{$\begin{array}{l}\text { Determined in PBS. }{ }^{b} \text { Determined in methanol. The molar } \\
\text { absorption coefficient, } \mathcal{E}_{\max } / \mathrm{mol}^{-1} \mathrm{dm}^{3} \mathrm{~cm}^{-1} \text {. }{ }^{d} \text { Values below the } \\
\text { detection limit of the setup. }{ }^{e} \mathrm{Absolute} \text { quantum yields of } \\
\text { photodecomposition. Average and standard deviations of the mean } \\
\text { are given. }\end{array}$} \\
\hline
\end{tabular}

The photouncaging ability was initially observed as a build-up of 4-methoxybenzoic acid in ${ }^{1} \mathrm{H}$ NMR spectra of $\mathbf{1 b}$ left in ambient light over several days (Figure S86). Intrigued by this, we followed irradiation of 1a in $\mathrm{CD}_{3} \mathrm{OD}$ with NIR light at $780 \mathrm{~nm}(\sim 40 \mathrm{~mW})$ by ${ }^{1} \mathrm{H}$ and ${ }^{19} \mathrm{~F}$ NMR spectroscopies. The irradiation was accompanied by disappearance of the signals of the caged 4-fluorobenzoic acid $\left(\delta_{\mathrm{F}} \sim-107.1 \mathrm{ppm}\right)$ and a concurrent emergence of the signals corresponding to the free acid ( $\delta_{\mathrm{F}} \sim-108.7 \mathrm{ppm}$; Figure $\left.4 \mathrm{~A}\right)$, whereas a sample left in the dark showed no changes (Figure 4A), demonstrating that the release is of photochemical origin. The process was found to be complete within $16 \mathrm{~h}$ despite high concentration $(\sim 3 \mathrm{mM})$ of $\mathbf{1 a}$.

We subsequently followed the photouncaging process by UV-vis absorption spectroscopy. Irradiation of 1a-b,1e $\left(\lambda_{\text {ir }} \sim 780 \mathrm{~nm} ; \sim 50 \mathrm{~mW} \mathrm{~cm}^{-2}\right)$ or $\mathbf{1} \mathbf{c}-\mathbf{d}\left(\lambda_{\text {irr }} \sim 820 \mathrm{~nm} ; \sim 40 \mathrm{~mW} \mathrm{~cm}^{-2}\right)$ in PBS (pH 7.4, $\left.10 \mathrm{mM}\right)$ was accompanied by depletion of the cyanine absorption band (Figure $2 \mathrm{~B}$ ) and concurrent weak increase of absorption at $\sim 400 \mathrm{~nm}$, attributed to the photobleaching products of cyanine scaffold. ${ }^{33}$ The photolysis was fully complete within 15-30 min (Figure 3C). The absolute quantum yields of photodecomposition ( $\left.\Phi_{\mathrm{dec}}\right)$ are summarized in Table 1 and were found to be comparable to the first generation of BODIPY photocages which, however, operate at wavelengths blue-shifted by $>100 \mathrm{~nm} .{ }^{26}$

To follow the photouncaging process in time, we prepared 1c containing caged fluorescent coumarinderived acid 3c, a potent inhibitor of lactate influx in various cancer cell lines. ${ }^{34}$ The coumarin residue in photocage $1 \mathrm{c}$ displays only weak fluorescence signal $\left(\lambda_{\mathrm{exc}}=420 \mathrm{~nm}\right)$ due to quenching by energy transfer to the dye. Irradiation of $\mathbf{1 c}$ at $820 \mathrm{~nm}$ led to a rapid decrease (within $10 \mathrm{~min}$ ) of absorption at $808 \mathrm{~nm}$ and a simultaneous increase of coumarin fluorescence intensity ( $\sim 6$-fold) as a consequence of the release of $\mathbf{3 c}$ (Figure 3D; Figure S83). The control samples left in the dark showed no increase of fluorescence intensity. 

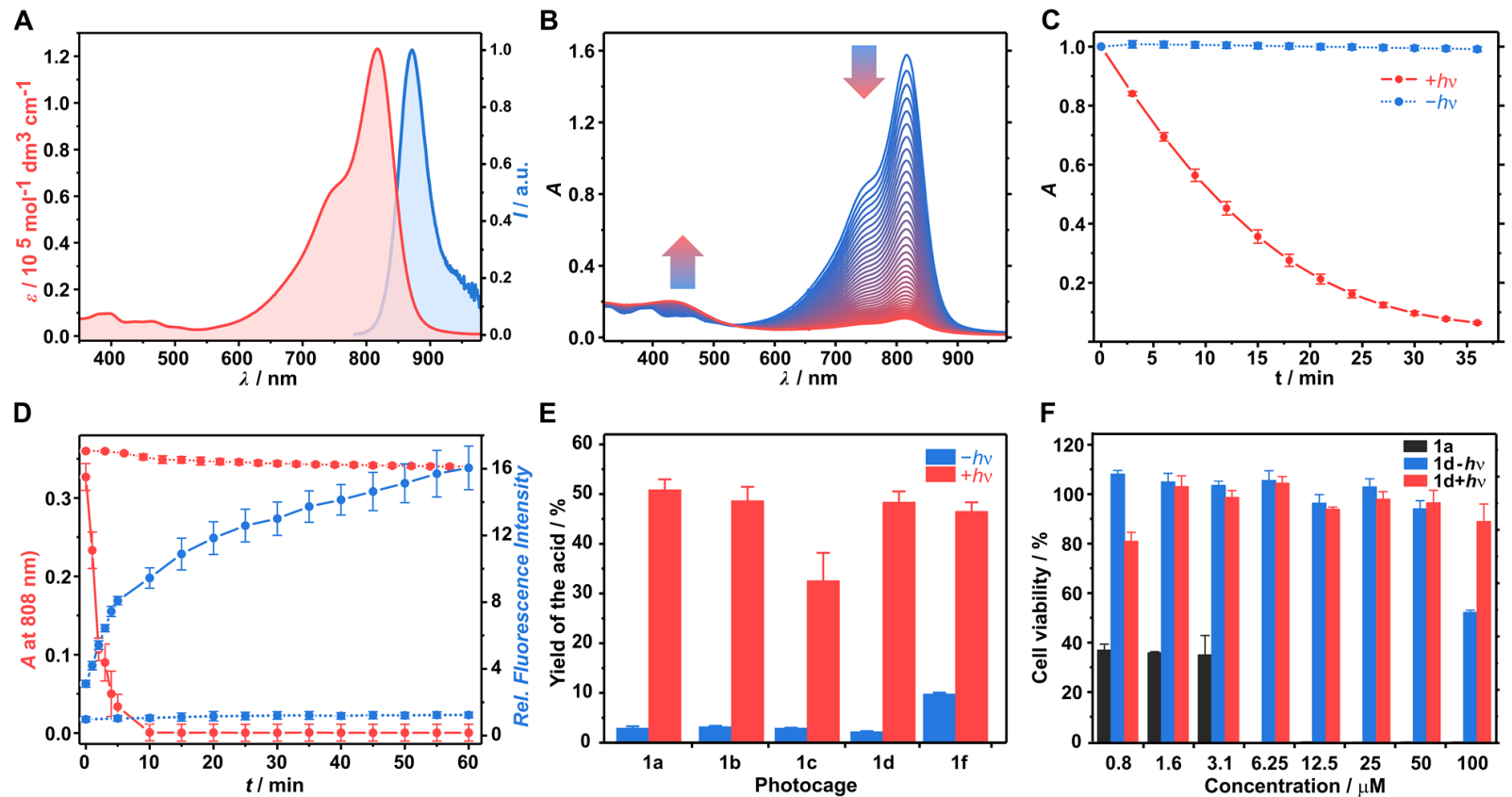

Figure 3. (A) Absorption (red) and emission (blue) spectra of 1d in PBS and methanol, respectively. (B) Irradiation of $1 \mathrm{~b}$ in PBS at at $820 \mathrm{~nm}$ followed by UV-vis spectroscopy every $60 \mathrm{~s}$ (blue to red). (C) Kinetic trace of the absorption at $817 \mathrm{~nm}$ upon irradiation of $\mathbf{1 d}$ at $820 \mathrm{~nm}$ (red) and in the dark (blue). (D) Absorption (red, $\lambda=808 \mathrm{~nm}$ ) and fluorescence (blue, $\lambda_{\mathrm{exc}}=390 \mathrm{~nm}$ ) traces of 1c with (solid) or without (dotted) irradiation in PBS at $820 \mathrm{~nm}$. (E) Yields of the acids released upon irradiation (red) and in the dark (blue) determined by HPLC. (F) Cell-viability of HeLa cells in the presence of 1a (black), 1d (blue) and photoproducts of $\mathbf{1 d}$ (red). Average of three experiments and standard deviations of the mean are given.

As the next step, we quantified the chemical yield of carboxylic acids released upon irradiation using HPLC (Figure 3E). The solutions of $1 \mathbf{a}-\mathbf{f}$ were irradiated and subsequently incubated at $37^{\circ} \mathrm{C}$ for $2 \mathrm{~h}$, whereas the control experiments were incubated in dark at $37{ }^{\circ} \mathrm{C}$ for $2 \mathrm{~h}$. The chemical yields of the released carboxylic acids were found to be generally $\sim 50 \%$, comparable to the existing NIR photocages. ${ }^{16,35}$ The control experiments incubated in the dark showed only a negligible release $(<3 \%)$.

Given the expected biological applications of the photocages, we evaluated the systemic toxicity of $\mathbf{1 a}$ and 1d on HeLa cells. While photocage $1 \mathbf{a}$ was found to be relatively toxic $\left(\mathrm{IC}_{50}<5 \mu \mathrm{M}\right)$ in cell viability assays, analogue 1d with appended solubilizing sulfonate groups and its corresponding photoproducts produced by exhaustive irradiation displayed no toxicity after $24 \mathrm{~h}$ exposure to concentrations as high as $50 \mu \mathrm{M}$ (Figure 3F). Such distinct effect of the $N$-substituents on the cytotoxicity of cyanine has been previously observed. ${ }^{36}$ Nevertheless, minimal intrinsic toxicity of $\mathbf{1 d}$ and its photoproducts reinforce the potential of water-soluble analogues of our photocages for applications in biological settings. 

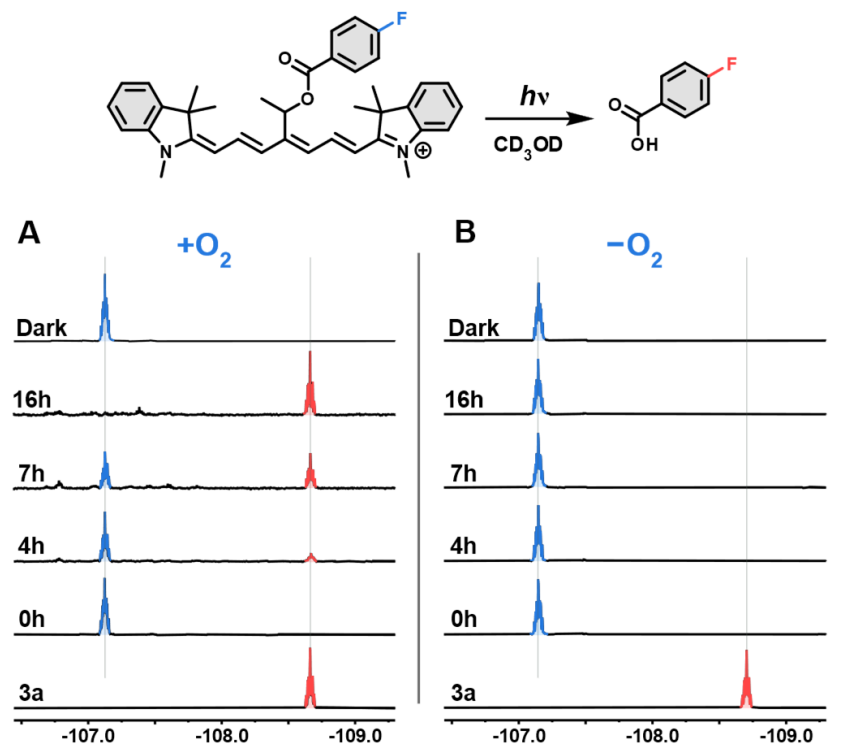

Figure 4. Photouncaging of $3 a$ upon irradiation at $780 \mathrm{~nm}$ followed by ${ }^{19} \mathrm{~F}$ NMR spectroscopy in the presence (A) and absence (B) of oxygen.

We hypothesized two plausible mechanistic explanations for the photouncaging process (Figure 5) - a selfsensitized photooxidation of the cyanine backbone by singlet oxygen and subsequent dark solvolysis of the photoproducts (Pathway A), or a direct light-initiated heterolysis with concurrent departure of the leaving group (Pathway B). Photooxdiation is the primary photobleaching pathway of cyanines that is well documented and proceeds via addition of photogenerated singlet oxygen $\left({ }^{1} \mathrm{O}_{2}\right)$ on the polyene backbone. ${ }^{33}$ These two pathways can be distinguished by the presence of oxygen. Since Pathway A directly relies on the reactivity of ${ }^{1} \mathrm{O}_{2}$, it will be halted in its absence. On the contrary, photoheterolysis B is expected to occur from the triplet excited state,$^{24}$ and its efficacy should therefore increase in the absence of oxygen as the productive excited state is quenched. Our experiments revealed that $\mathbf{1 a}$ is stable to irradiation in degassed $\mathrm{CD}_{3} \mathrm{OD}$ and no release of $\mathbf{3 a}$ was detected by ${ }^{1} \mathrm{H}$ or ${ }^{19} \mathrm{~F}$ NMR spectroscopies (Figure $4 \mathrm{~B}$ ). We also identified ketone 7 as a product of photooxidation ${ }^{16,33}$ of $\mathbf{1 a}$ irradiated under ambient conditions (Figure S87). Analogous photouncaging reactivity was also observed when 1a was irradiated at $590 \mathrm{~nm}$ in the presence of methylene blue as an auxiliary singlet oxygen generator (Figure S90-91). Furthermore, saturating methanolic solution of 1a with argon led to $\sim 3$-fold decrease of $\Phi_{\text {dec. }}$. These observations demonstrate the central role of oxygen in the observed photouncaging and constitute compulsive evidence for the selfsensitized photooxygenation pathway A. This notion is corroborated by the time profile of $\mathbf{3 c}$ uncaging in Figure 3D. The release continued even after the cyanine absorption was fully depleted, suggesting that irradiation is necessary to disrupt the cyanine scaffold and the subsequent step that culminates in the release of the acid occurs in the dark. The photouncaging proceeds also from $1 \mathrm{e}$ bearing $t \mathrm{Bu}$ at the reaction center, suggesting that hydrogens of the methyl group 1a-d are not involved in the release of $\mathbf{3 a}$ from $\mathbf{8}$.

We reasoned that stabilization of the putative carbocation formed in pathway B could result in favoring the photoheterolysis. Hence, we introduced an additional methyl group at the reaction center in $\mathbf{1 f}$. The stability of homologue $\mathbf{1 f}$ was compromised, complicating its purification and also manifesting in faster hydrolysis in the dark ( $\sim 10 \%$, Figure 3E). However, we found the derivative $\mathbf{1 f}$ to be the only photocage that gave rise to a new signal $\left(\delta_{\mathrm{F}} \sim-108.4 \mathrm{ppm}\right)$ in ${ }^{19} \mathrm{~F}$ NMR spectra when irradiation under oxygen-free conditions (Figure 
S93-95), whereas no such change was observed for $1 \mathbf{f}$ in dark. However, the chemical shift of the photoproduct is shifted from that of $\mathbf{3 a}\left(\delta_{\mathrm{F}} \sim-108.7 \mathrm{ppm}\right)$ by $0.3 \mathrm{ppm}$. We hypothesized this could be caused by small changes of $\mathrm{pH}$ in the solution, which is known to have a dramatic effect on ${ }^{19} \mathrm{~F}$ NMR shifts. ${ }^{37}$ Spiking the sample with 3a led to disappearance of this signal, whereas other signals remained unaffected (Figure S96). In conjunction with the compromised thermal stability, we consider the observation of direct photoheterolysis in $\mathbf{1 f}$ to be not fully conclusive and this concept will be investigated further in a subsequent work.

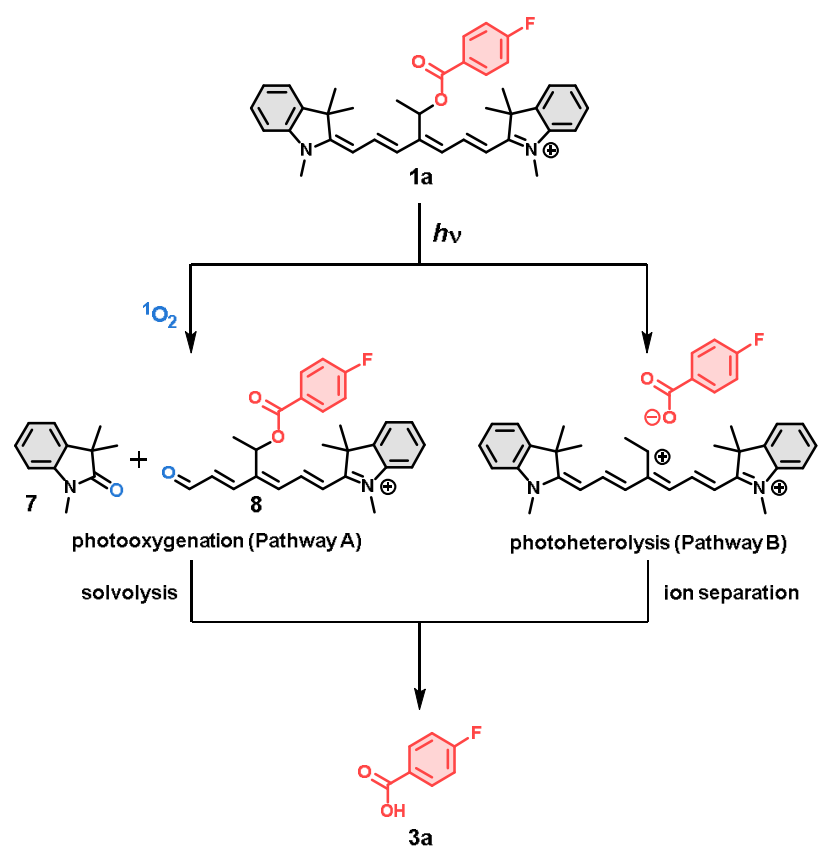

Figure 5. Plausible mechanisms of the photouncaging process.

\section{Conclusions}

In conclusion, we report a class of photocages that can be synthesized in three steps on a multigram scale and release carboxylic acids upon irradiation with NIR light up to $820 \mathrm{~nm}$ in aqueous media. The photouncaging process was examined independently using ${ }^{1} \mathrm{H},{ }^{19} \mathrm{~F}$ NMR and emission spectroscopies, and HPLC analysis. The preliminary experiments currently underway in our laboratory suggest that this design is broadly applicable and can be extended towards the release of alcohols and amines. In combination with the facile synthesis and modularity of the cyanine scaffold, our readily available photocages constitute an attractive alternative to the existing NIR-uncaging systems. We are convinced that they will make photoactivation using tissue-penetrating light accessible also to non-specialist outside the photochemistry field, similar to the success of $o$-nitrobenzyl in the past.

\section{Author Contributions}

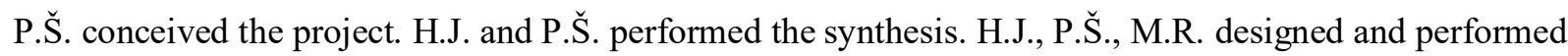
the photochemical experiments. H.J. performed the biological experiments. All authors co-wrote the manuscript. 


\section{Author Information}

\section{Corresponding Authors}

* peter.stacko@uzh.ch

\section{Notes}

The authors declare no competing interests.

\section{Acknowledgment}

We gratefully acknowledge Swiss National Science Foundation (SNSF, P.Š/PZ00P2_193425) and the Department of Chemistry, University of Zurich (Legerlotz Stiftung and prof. Hans Schmidt Stiftung) for funding of this research project. We would like to thank prof. Cristina Nevado, prof. Karl Gademann and prof. Michal Juríček (University of Zurich) for the generous support of our research. We thank prof. Jason P. Holland for access to their HPLC, Dr. Katherine Gosselé for assistance with cell-viability assays, and prof. Tomáš Šolomek and prof. Petr Klán for fruitful suggestions.

\section{References}

(1) Klán, P.; Šolomek, T.; Bochet, C. G.; Blanc, A.; Givens, R.; Rubina, M.; Popik, V.; Kostikov, A.; Wirz, J. Photoremovable Protecting Groups in Chemistry and Biology: Reaction Mechanisms and Efficacy. Chem. Rev. 2013, 113, 119-191.

(2) Lawrence, D. S. The Preparation and in Vivo Applications of Caged Peptides and Proteins. Curr. Opin. Chem. Biol. 2005, 9, 570-575.

(3) Zhao, J.; Zhao, J.; Lin, S.; Huang, Y.; Chen, P. R. Mechanism-Based Design of a Photoactivatable Firefly Luciferase. J. Am. Chem. Soc. 2013, 135, 7410-7413.

(4) Kaplan, J. H.; Forbush, B.; Hoffman, J. F. Rapid Photolytic Release of Adenosine 5'-Triphosphate from a Protected Analogue: Utilization by the Na:K Pump of Human Red Blood Cell Ghosts. Biochemistry 1978, 17, 1929-1935.

(5) Walker, J. W.; Reid, G. P.; McCray, J. A.; Trentham, D. R. Photolabile 1-(2-Nitrophenyl)Ethyl Phosphate Esters of Adenine Nucleotide Analogues. Synthesis and Mechanism of Photolysis. $J$. Am. Chem. Soc. 1988, 110, 7170-7177.

(6) Lin, Q.; Huang, Q.; Li, C.; Bao, C.; Liu, Z.; Li, F.; Zhu, L. Anticancer Drug Release from a Mesoporous Silica Based Nanophotocage Regulated by Either a One- or Two-Photon Process. J. Am. Chem. Soc. 2010, 132, 10645-10647.

(7) Liu, M.; Meng, J.; Bao, W.; Liu, S.; Wei, W.; Ma, G.; Tian, Z. Single-Chromophore-Based Therapeutic Agent Enables Green-Light-Triggered Chemotherapy and Simultaneous Photodynamic Therapy to Cancer Cells. ACS Appl. Bio Mater. 2019, 2, 3068-3076.

(8) Mayer, G.; Hechel, A. Biologically Active Molecules with a "Light Switch." Angew. Chem. Int. Ed. 2006, 45, 4900-4921.

(9) Ellis-Davies, G. C. R. Caged Compounds: Photorelease Technology for Control of Cellular Chemistry and Physiology. Nat. Methods 2007, 4, 619-628.

(10) Kramer, R. H.; Chambers, J. J.; Trauner, D. Photochemical Tools for Remote Control of Ion Channels in Excitable Cells. Nat. Chem. Biol. 2005, 1, 360-365.

(11) Weissleder, R. A Clearer Vision for in Vivo Imaging. Nat. Biotechnol. 2001, 19, 316-317.

(12) Lim, Y. T.; Kim, S.; Nakayama, A.; Stott, N. E.; Bawendi, M. G.; Frangioni, J. V. Selection of Quantum Dot Wavelengths for Biomedical Assays and Imaging. Mol. Imaging 2003, 2, 50-64.

(13) Weinstain, R.; Slanina, T.; Kand, D.; Klán, P. Visible-to-NIR-Light Activated Release: From Small Molecules to Nanomaterials. Chem. Rev. 2020.

(14) Štacko, P.; Šolomek, T. Photoremovable Protecting Groups: Across the Light Spectrum to near- 
Infrared Absorbing Photocages. Chimia. 2021, 75, 873-881.

(15) Anderson, E. D.; Gorka, A. P.; Schnermann, M. J. Near-Infrared Uncaging or Photosensitizing Dictated by Oxygen Tension. Nat. Commun. 2016, 7, 13378.

(16) Gorka, A. P.; Nani, R. R.; Zhu, J.; Mackem, S.; Schnermann, M. J. A Near-IR Uncaging Strategy Based on Cyanine Photochemistry. J. Am. Chem. Soc. 2014, 136, 14153-14159.

(17) Gorka, A. P.; Nani, R. R.; Schnermann, M. J. Harnessing Cyanine Reactivity for Optical Imaging and Drug Delivery. Acc. Chem. Res. 2018, 51, 3226-3235.

(18) Yamamoto, T.; Caldwell, D. R.; Gandioso, A.; Schnermann, M. J. A Cyanine Photooxidation/ $\beta-$ Elimination Sequence Enables Near-Infrared Uncaging of Aryl Amine Payloads. Photochem. Photobiol. 2019, 95, 951-958.

(19) Nani, R. R.; Gorka, A. P.; Nagaya, T.; Kobayashi, H.; Schnermann, M. J. Near-IR Light-Mediated Cleavage of Antibody-Drug Conjugates Using Cyanine Photocages. Angew. Chem. Int. Ed. 2015, $54,13635-13638$.

(20) Nani, R. R.; Gorka, A. P.; Nagaya, T.; Yamamoto, T.; Ivanic, J.; Kobayashi, H.; Schnermann, M. J. In Vivo Activation of Duocarmycin-Antibody Conjugates by Near-Infrared Light. ACS Cent. Sci. 2017, 3, 329-337.

(21) Black, C. E.; Zhou, E.; DeAngelo, C. M.; Asante, I.; Louie, S. G.; Petasis, N. A.; Humayun, M. S. Cyanine Nanocages Activated by Near-Infrared Light for the Targeted Treatment of Traumatic Brain Injury. Front. Chem. 2020, 8, 769.

(22) Goswami, P. P.; Syed, A.; Beck, C. L.; Albright, T. R.; Mahoney, K. M.; Unash, R.; Smith, E. A.; Winter, A. H. BODIPY-Derived Photoremovable Protecting Groups Unmasked with Green Light. J. Am. Chem. Soc. 2015, 137, 3783-3786.

(23) Rubinstein, N.; Liu, P.; Miller, E. W.; Weinstain, R. Meso-Methylhydroxy BODIPY: A Scaffold for Photo-Labile Protecting Groups. Chem. Commun. 2015, 51, 6369-6372.

(24) Slanina, T.; Shrestha, P.; Palao, E.; Kand, D.; Peterson, J. A.; Dutton, A. S.; Rubinstein, N.; Weinstain, R.; Winter, A. H.; Klán, P. In Search of the Perfect Photocage: Structure-Reactivity Relationships in Meso-Methyl BODIPY Photoremovable Protecting Groups. J. Am. Chem. Soc. 2017, 139, 15168-15175.

(25) Peterson, J. A.; Fischer, L. J.; Gehrmann, E. J.; Shrestha, P.; Yuan, D.; Wijesooriya, C. S.; Smith, E. A.; Winter, A. H. Direct Photorelease of Alcohols from Boron-Alkylated BODIPY Photocages. J. Org. Chem. 2020, 85, 5712-5717.

(26) Peterson, J. A.; Wijesooriya, C.; Gehrmann, E. J.; Mahoney, K. M.; Goswami, P. P.; Albright, T. R.; Syed, A.; Dutton, A. S.; Smith, E. A.; Winter, A. H. Family of BODIPY Photocages Cleaved by Single Photons of Visible/Near-Infrared Light. J. Am. Chem. Soc. 2018, 140, 7343-7346.

(27) Sitkowska, K.; Hoes, M. F.; Lerch, M. M.; Lameijer, L. N.; Van Der Meer, P.; Szymański, W.; Feringa, B. L. Red-Light-Sensitive BODIPY Photoprotecting Groups for Amines and Their Biological Application in Controlling Heart Rhythm. Chem. Commun. 2020, 56, 5480-5483.

(28) Shrestha, P.; Dissanayake, K. C.; Gehrmann, E. J.; Wijesooriya, C. S.; Mukhopadhyay, A.; Smith, E. A.; Winter, A. H. Efficient Far-Red/Near-IR Absorbing BODIPY Photocages by Blocking Unproductive Conical Intersections. J. Am. Chem. Soc. 2020, 142, 15505-15512.

(29) Zimmerman, H. E.; Somasekhara, S. Mechanistic Organic Photochemistry. III. Excited State Solvolyses. J. Am. Chem. Soc. 1963, 85, 922-927.

(30) Šolomek, T.; Wirz, J.; Klán, P. Searching for Improved Photoreleasing Abilities of Organic Molecules. Acc. Chem. Res. 2015, 48, 3064-3072.

(31) Štacková, L.; Štacko, P.; Klán, P. Approach to a Substituted Heptamethine Cyanine Chain by the Ring Opening of Zincke Salts. J. Am. Chem. Soc. 2019, 141, 7155-7162.

(32) Štacková, L.; Muchová, E.; Russo, M.; Slavíček, P.; Štacko, P.; Klán, P. Deciphering the Structure-Property Relations in Substituted Heptamethine Cyanines. J. Org. Chem. 2020, 85, 9776-9790.

(33) Nani, R. R.; Kelley, J. A.; Ivanic, J.; Schnermann, M. J. Reactive Species Involved in the Regioselective Photooxidation of Heptamethine Cyanines. Chem. Sci. 2015, 6, 6556-6563. 
(34) Draoui, N.; Schicke, O.; Seront, E.; Bouzin, C.; Sonveaux, P.; Riant, O.; Feron, O. Antitumor Activity of 7-Aminocarboxycoumarin Derivatives, a New Class of Potent Inhibitors of Lactate Influx but Not Efflux. Mol. Cancer Ther. 2014, 13, 1410-1418.

(35) Štacko, P.; Muchová, L.; Vítek, L.; Klán, P. Visible to NIR Light Photoactivation of Hydrogen Sulfide for Biological Targeting. Org. Lett. 2018, 20, 4907-4911.

(36) Atkinson, K. M.; Morsby, J. J.; Kommidi, S. S. R.; Smith, B. D. Generalizable Synthesis of Bioresponsive Near-Infrared Fluorescent Probes: Sulfonated Heptamethine Cyanine Prototype for Imaging Cell Hypoxia. Org. Biomol. Chem. 2021, 19, 4100-4106.

(37) Boiadjiev, S. E.; Lightner, D. A. Carboxylic Acid Ionization Constants by 19F NMR Spectroscopy. J. Phys. Org. Chem. 1999, 12, 751-757. 